\title{
Bursa Ili Memeli (Classis: Mammalia) Faunası ve Türlerin Koruma Statüleri
}

\author{
Serdar Gözütok ${ }^{*}$
}

Abant İzzet Baysal Üniversitesi, Ziraat ve Doğa Bilimleri Fakültesi, Yaban Hayatı Ekolojisi ve Yönetimi Bölümü, Bolu

\author{
Geliş tarihi (Received): 12.06.2017Kabul tarihi (Accepted): 19.07.2017
}

\begin{abstract}
Anahtar kelimeler:
Özet. Bursa ilinde, 2014-2015 yılları arasında Bursa ili memeli faunası veri tabanına katkı Memeliler, fauna, Bursa, koruma durumları sağlamak amacıyla arazi çalışmaları ve literatür incelemeleri yapılmıştır. Arazi çalışmaları canlı yakalama kapanları ve fotokapanların kurulması yanında hayvanlara ait doğrudan yapılan gözlemler, yuva, dışkı, ayak izi, baykuş pelletlerindeki hayvanlara ait vücut parçalarının toplanması şeklinde yapılmıştır. Elde edilen türlerin korunma durumları IUCN, BERN, CITES, MAKK bakımından incelenmiş ve güncel korunma statüleri kaydedilmiştir. Tespit edilen alanlardaki türler için koruma önerileri sunulmuştur. Çalışmadan elde edilen sonuçlara göre, Memeli sınıfına ait altı takıma mensup 23 türün varlığı tespit edilmiştir. Yaygınlık bakımından öne çıkan türlerin Sus scrofa Linnaeus, 1758, Erinaceus concolor

*Sorumlu yazar serdargozutok@ibu.edu.tr

Martin, 1837, Apodemus flavicollis (Melchior, 1834), Rattus rattus (Linnaeus, 1758), Sciurus anomalus Gmelin, 1778 olduğu anlaşılmıştır. Sadağı Kanyonu'nda tespit edilen Lutra lutra (Linnaeus,1758) (Su samuru) il için yeni bir yayılış kaydı olarak önem arz etmektedir.
\end{abstract}

\section{Mammalian Fauna of Bursa Province and Conversation Status of Species (Classis: Mammalia)}

\section{Keywords:}

Mammals, fauna, Bursa, conservation status

\begin{abstract}
Field studies as well as literature reviews conducted to contribute to the data base on mammalian fauna of Bursa province between 2014-2015. During the field studies, information collected from live traps and wildlife camera traps along with the direct animal observations, nest, feces, footprint and analysis of body parts of animals in Owl pellets. The conservation status of the obtained species was examined in terms of IUCN, BERN, CITES, MAKK and actual protection status were recorded. Protection recommendations for the species in the identified areas are provided. The study revealed that 23 species belonging to the Classis Mammalia were present in the province. The most prevalent species were Sus scrofa Linnaeus, 1758, Erinaceus concolor Martin, 1837, Apodemus flavicollis (Melchior, 1834), Rattus rattus (Linnaeus, 1758), Sciurus anomalus Gmelin, 1778. It was also important that Lutra lutra (Linnaeus, 1758) (The Eurasian otter) detected in Sadağı Canyon was a new distribution record for the province.
\end{abstract}




\section{GíRiş}

Mammalia sınıfı Dünya'da 29 takım, 153 familya, 1229 cins ve 5416 tür ile temsil edilmektedir (Musser and Carleton, 2005). Ülkemizde sekiz takıma ait 161 memeli türü bulunmaktadır (Yiğit ve ark., 2006). Bursa ili ve yakın çevresinde araştırıcılar tarafından Memeli sınıfının üyelerinin yayılış durumlarını, taksonomik ve ekolojik özelliklerini konu alan çok sayıda çalışma yapılmıştır. (Neuhäuser 1936; Kahmann ve Çağlar 1960; Çağlar 1961; Çağlar 1965; Osborn 1965a; Kumerloeve 1967; Çağlar, 1968; Spitzenberger 1968; Spitzenberger 1970; Felten et al., 1971; Doğramacı 1974; Mursaloğlu 1976; Felten et al., 1977; Kıvanç 1990; Vohralík 1991; Albayrak 1993; Kefelioğlu 1995; Zima and Macholán 1995; Kefelioğlu ve Gençoğlu 1996; Macholán and Zima 1997; Yarar ve Magnin 1997; Benda and Horáček 1998; Kryštufek 1999; Tez 2000; Kryštufek and Vohralík 2001; Sert ve ark., 2001; Karataş and Sözen 2004; Kryštufek and Vohralík 2005; Matur and Sözen 2005; Yiğit ve ark., 2006; Kryštufek and Vohralík 2009). Bursa ilinde 49 adet memeli türünün yaşadığı kaydedilmiştir (ÇED 2016).

Bu araştırmada Bursa'da yapılan arazi çalışmaları sonucunda elde edilen verilerle ilin memeli faunasının güncel durumunun ve koruma statülerinin anlaşılmasına katkı yapılması amaçlanmıştır.

\section{MATERYAL VE METOD}

Bursa, Türkiye'nin kuzeybatısında, Marmara ve Ege Bölgelerinde, $28^{\circ} 10^{\prime}$ ve $30^{\circ} 10^{\prime}$ kuzey enlemleriyle, $40^{\circ}$ $40^{\prime}$ ve $39^{\circ} 35^{\prime}$ doğu boylamları arasında yer almaktadır. Eskiden Keşiş Dağı (Olympos Mysios) denilen Uludağ'ın (2543 m) kuzeybatı eteklerinde, şehrin adını taşıyan ovanın güney kenarında yer alır (Şekil 1). İlin toplam orman alanı 484.067 ha'dır (IÇDR 2011). Bursa'nın meteorolojik verileri Çizelge 1'de gösterilmektedir (MGM 2017).

Bursa ili doğal çevrelerinde memeli türlerinin tespitine yönelik 2014 ve 2015 yılları arasında doğrudan ve dolaylı gözlemler yapılmış bunlara ek olarak literatür incelemesi yapılmışıı. Ayrıca yerel halk ile yapılan görüşmelerle türlerin yayılışları hakkında bilgiler edinilmiştir. Arazi çalışmalarında memeli hayvanlara ait yuva, dışkı, kıl, ayak izi, eşeleme, ses gibi dolaylı biyolojik veriler yanında canlı yakalama kapanları ile yakalanan türlerin resimleri, fotokapanlar sayesinde elde edilen görüntüler, ölü olarak karşılaşılan örnekler ve baykuş peletlerinden tespit edilen türler de kaydedilmiştir. Canlı yakalama kapanlarına fıstıkla çiğnenmiş ekmek konulmuş gün batımına yakın saatlerde kurulmuş gün doğarken toplanarak örnekler incelenmiştir. Fotokapanlar hayvanların muhtemel yaşam alanları, geçiş noktaları, patikalar, yuva yakınları gibi noktalara yerleştirilmiştir (Şekil 2).

Tespit edilen türlerin IUCN (2017), (The International Union for Conservation of Nature), BERN (1979), (Conservation of European Wildlife and Natural Habitats), CITES, (The Convention on International Trade in Endangered Species of Wild Fauna and Flora), MAKK (2016) (Merkez Av Komisyonu Kararları)'na göre korunma durumları tablolarda verilmiştir. IUCN'e göre türün bulunduğu statüyü ifade eden kısaltmalar çizelge altında açıklanmıştır. Literatür kayıtları incelenerek diğer araştırıcıların yaptığı araştırmaların verileri değerlendirilmiştir.

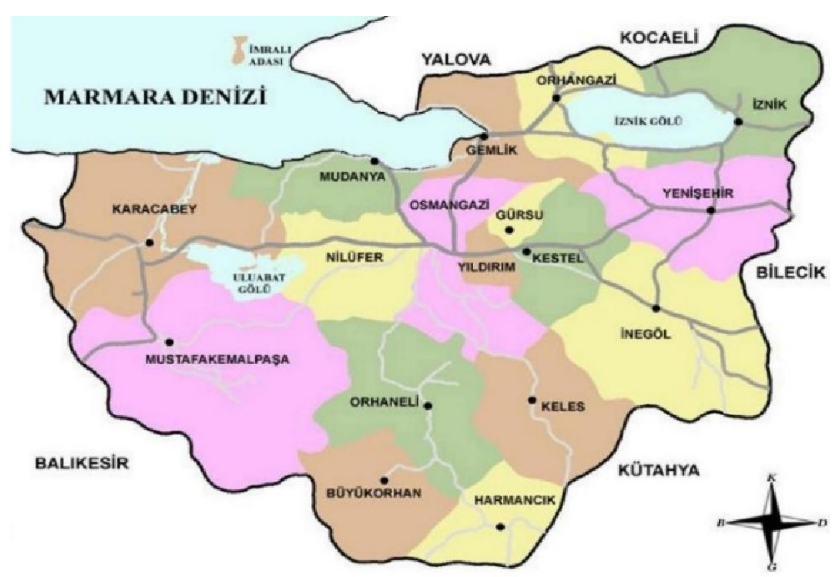

Şekil 1. Bursa ili ve ilçelerini gösteren harita. Figure 1. Map of Bursa province and districts.

\section{BULGULAR VE TARTIŞMA}

Bursa ilinin bulunduğu bölgede diğer araştırıcılar tarafından elde edilen tür kayıtları Çizelge $2^{\prime}$ de gösterilmektedir.

Toplanan verilerin analizi sonucunda Bursa'dan Mammalia sınıfının altı takımına ait 23 tür tespit edilmiştir. Elde edilen verilere göre Bursa'da arazide tespit edilen türlerin koruma statüleri ve bulundukları lokaliteler aşağıda verilmiştir (Çizelge 3).

Arazi çalışmaları sonucunda, Carnivora takımına ait; Canis aureus (Çakal), Canis lupus (Kurt), Felis silvestris (Yaban kedisi), Lutra lutra (Su samuru), Martes foina (Kaya sansarı), Meles meles (Porsuk), Mustela nivalis (Gelincik), Ursus arctos (Boz ayı), Vulpes vulpes (Kızıl tilki), Cetartiodactyla takımına ait; Capreolus capreolus (Karaca), Sus scrofa (Yaban Domuzu), Chiroptera takımına ait; Pipistrellus pipistrellus (Cüce yarasa), Rhinolophus hipposideros (Küçük Nalburunlu yarasa), Eulipotyphila takımına ait; Crocidura leucodon (Beyazdişli böcekçil), Erinaceus concolor (Kirpi), 
Lagomorpha takımına ait; Lepus europaeus (Yaban tavşanı), Rodentia takımına ait; Apodemus flavicollis, Mus musculus (Ev faresi), Nannospalax xanthodon (Körfare), Rattus rattus (Sıçan), Sciurus anomalus (Anadolu sincabı), Microtus levis, Muscardinus avellanarius (Fındık faresi) tespit edilmiştir. Bursa ilinde arazi çalışmalarında tespit edilen türlerden bir tür, Lutra lutra (Su samuru) IUCN'e göre NT kategorisinde bulunmaktadır.

Çizelge 1. Bursa ili meteorolojik verileri (1926-2016).

Table 1. Meteorological data of Bursa province (1926-2016).

\begin{tabular}{|c|c|c|c|c|c|c|c|c|c|c|c|c|c|}
\hline \multicolumn{14}{|c|}{ Uzun Yıllar İçinde Gerçekleşen Ortalama Değerler } \\
\hline & Ocak & Şubat & Mart & Nisan & Mayıs & Haziran & Temmuz & Ağustos & Eylül & Ekim & Kasım & Aralık & Yıllık \\
\hline Ort. Sıcaklık $\left({ }^{\circ} \mathrm{C}\right)$ & 5.3 & 6.1 & 8.3 & 12.9 & 17.6 & 22.0 & 24.5 & 24.2 & 20.1 & 15.4 & 10.9 & 7.3 & 14.6 \\
\hline $\begin{array}{l}\text { Ort. En Yüksek } \\
\text { Sıcaklık }\left({ }^{\circ} \mathrm{C}\right)\end{array}$ & 9.4 & 10.7 & 13.7 & 18.9 & 23.8 & 28.3 & 30.8 & 31.0 & 27.2 & 22.0 & 16.6 & 11.5 & 20.3 \\
\hline $\begin{array}{l}\text { Ort. En Düşük } \\
\text { Sıcaklık }\left({ }^{\circ} \mathrm{C}\right) \\
\text { Aylık Toplam }\end{array}$ & 1.7 & 2.1 & 3.5 & 7.2 & 11.3 & 14.8 & 17.1 & 17.1 & 13.6 & 10.1 & 6.4 & 3.5 & 9.0 \\
\hline $\begin{array}{l}\text { Yağış Miktarı } \\
\text { Ort. }\left(k \text { m }^{-2}\right)\end{array}$ & 89.1 & 76.7 & 70.1 & 63.0 & 49.2 & 33.3 & 21.6 & 16.6 & 42.0 & 66.8 & 78.4 & 100.7 & 707.5 \\
\hline \multicolumn{14}{|c|}{ Uzun Yıllar İçinde Gerçekleşen En Yüksek ve En Düşük Değerler } \\
\hline $\begin{array}{l}\text { En Yüksek } \\
\text { Sıcaklık }\left({ }^{\circ} \mathrm{C}\right)\end{array}$ & 23.8 & 26.9 & 32.5 & 36.2 & 38.2 & 41.3 & 43.8 & 42.6 & 40.1 & 37.3 & 34.0 & 27.3 & 43.8 \\
\hline $\begin{array}{l}\text { En Düşük } \\
\text { Sıcaklık }\left({ }^{\circ} \mathrm{C}\right) \\
\end{array}$ & -20.5 & -25.7 & -10.5 & -4.2 & 0.8 & 4.0 & 8.3 & 7.6 & 3.3 & -1.0 & -8.4 & -17.9 & -25.7 \\
\hline
\end{tabular}
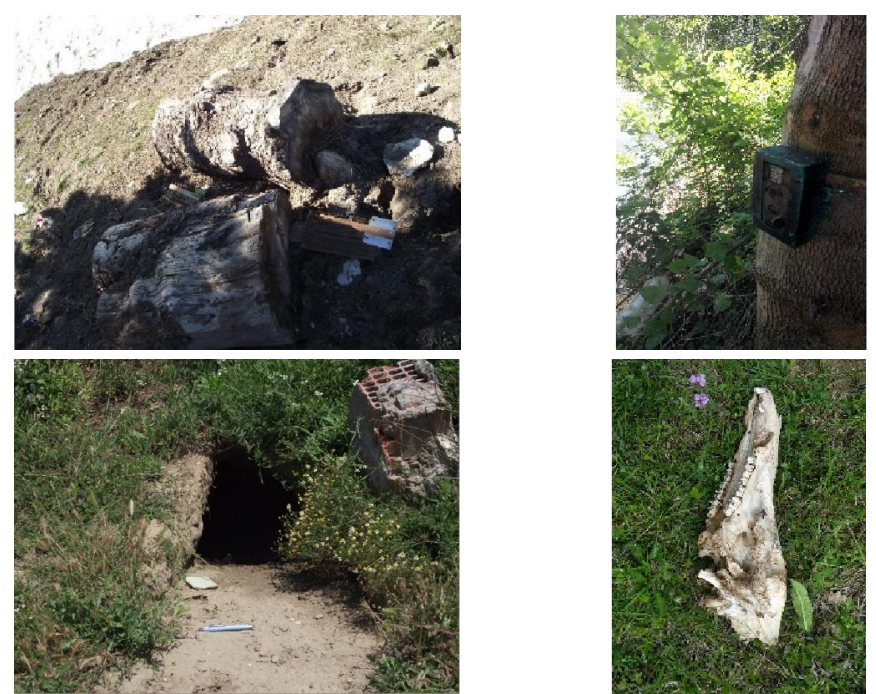

Çalışmaya ilişkin türlerin kaydedilebilen fotoğrafları aşağıda bulunmaktadır (Şekil 3, 4, 5 ve 6).

Elde edilen verilerle bölgeden daha önce araştırıcılara ait kayıtlar uyumluluk göstermektedir. Lutra lutra için verilen lokalite il için yeni bir yayılış kaydı olması bakımından türün korunması ve yapılacak planlama çalışmaları için fayda sağlayacaktır.

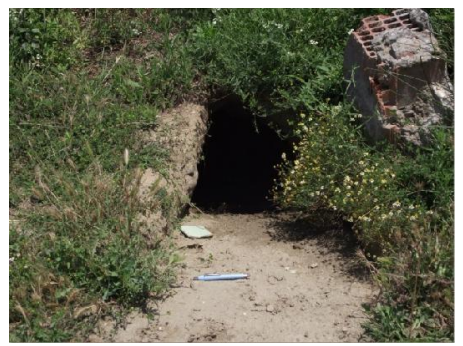

Şekil 2. Arazi çalışmalarında tür tespiti için kullanılan araç, gereç ve kanıtlar (Soldan sağa: Canlı yakalama kapanları, fotokapan, ayak izi, dışkı, yuva, vücut parçaları, baykuş peleti).

Figure 2. Tools, equipment and evidence used for species detection during field studies (From left to right: live traps, wildlife camera traps, footprint, feces, nest, body parts, owl pellets). 
Çizelge 2. Araştırma alanından kaydedilen memeli türlerinin yayılış kayıtları. Table 2. Distribution records of mammals species from research area.

\begin{tabular}{|c|c|c|c|}
\hline Takım & Familya & Tür & Literatür kaydı \\
\hline \multirow[t]{10}{*}{ Carnivora } & Canidae & Canis aureus Linnaeus, 1758 & Huş ve Göksel (1981) \\
\hline & & Vulpes vulpes (Linnaeus, 1758) & Huş ve Göksel (1981); Doğramacı (1989c) \\
\hline & Felidae & Lynx lynx (Linnaeus, 1758) & Kumerloeve (1967); Huş ve Göksel (1981) \\
\hline & & Felis chaus Schreber, 1777 & ÇED (2016) \\
\hline & & Felis silvestris Schreber, 1777 & ÇED (2016) \\
\hline & Mustelidae & Lutra lutra (Linnaeus, 1758) & Kumerloeve (1967) \\
\hline & & Martes foina (Erxleben, 1777) & Kumerloeve (1967) \\
\hline & & Meles meles (Linnaeus, 1758) & $\begin{array}{l}\text { Huş ve Göksel (1981); Doğramacı } \\
\text { (1989c); Kumerloeve (1967) }\end{array}$ \\
\hline & & Mustela nivalis (Linnaeus, 1766) & Kumerloeve (1967); Kasparek (1988) \\
\hline & Ursidae & Ursus arctos Linnaeus, 1758 & Kumerloeve (1967); Huş ve Göksel (1981) \\
\hline \multirow[t]{3}{*}{ Cetartiodactyla } & Cervidae & Capreolus capreolus (Linnaeus, 1758) & $\begin{array}{l}\text { Kumerloeve (1967); Yarar ve Magnin } \\
\text { (1997) }\end{array}$ \\
\hline & & Cervus elaphus Linnaeus, 1758 & Kumerloeve (1967) \\
\hline & Suidae & Sus scrofa Linnaeus, 1758 & Yarar ve Magnin (1997) \\
\hline \multirow[t]{13}{*}{ Chiroptera } & Miniopteridae & Miniopterus schreibersii (Kuhl, 1817) & $\begin{array}{l}\text { Çağlar (1965); Kıvanç (1990), Benda and } \\
\text { Horáček (1998); Karataş ve Sözen (2004) }\end{array}$ \\
\hline & Rhinolophidae & Rhinolophus blasii Peters, 1867 & Benda and Horáček (1998) \\
\hline & & Rhinolophus euryale Blasius, 1853 & ÇED (2016) \\
\hline & & $\begin{array}{ll}\text { Rhinolophus } & \text { ferrumequinum } \\
\text { (Schreber, 1774) } & \end{array}$ & $\begin{array}{l}\text { Çağlar (1965); Çağlar (1968); Felten et al. } \\
\text { (1977), Benda and Horáček (1998) }\end{array}$ \\
\hline & & $\begin{array}{l}\text { Rhinolophus hipposideros (Bechstein, } \\
\text { 1800) }\end{array}$ & $\begin{array}{l}\text { Çağlar (1961); Çağlar (1965); Çağlar } \\
\text { (1968) }\end{array}$ \\
\hline & Vespertilionidae & Myotis capaccinii (Bonaparte, 1837) & Kıvanç (1990) \\
\hline & & Myotis daubentonii (Kuhl, 1817) & Kıvanç (1990) \\
\hline & & Myotis myotis (Borkhausen, 1797) & Benda and Horáček (1998) \\
\hline & & Myotis mystacinus (Kuhl, 1817) & Kıvanç (1990) \\
\hline & & Myotis blythii (Tomes, 1857) & ÇED 2016 \\
\hline & & Nyctalus lasiopterus (Schreber, 1780) & Kahmann ve Çağlar (1960); Çağlar (1965) \\
\hline & & $\begin{array}{l}\text { Pipistrellus pipistrellus (Schreber, } \\
\text { 1774) } \\
\text { Pipistrellus kuhli (Kuhl, 1817) }\end{array}$ & $\begin{array}{l}\text { Albayrak (1993); Benda and Horáček } \\
\text { (1998); Kıvanç (1990) } \\
\text { ÇED (2016) }\end{array}$ \\
\hline & & Hypsugo savii (Bonaparte, 1837) & Kıvanç (1990) \\
\hline \multirow[t]{7}{*}{ Eulipotyphla } & Erinaceidae & Erinaceus concolor Martin, 1837 & Huş ve Göksel (1981) \\
\hline & Soricidae & Crocidura leucodon (Hermann, 1780) & $\begin{array}{l}\text { Spitzenberger (1970); Felten et al. } \\
\text { (1973); Şimşek (1979); Tez (2000); } \\
\text { Kryštufek and Vohralík (2001) }\end{array}$ \\
\hline & & Crocidura suaveolens (Pallas, 1811) & $\begin{array}{l}\text { Spitzenberger (1970); Şimşek (1979); } \\
\text { Şimşek (1980); Kefelioğlu ve Tez (1999); } \\
\text { Tez (2000); Kryštufek and Vohralík } \\
\text { (2001); Sert et al. (2001) }\end{array}$ \\
\hline & & Neomys anomalus Cabrera, 1907 & $\begin{array}{l}\text { Kryštufek, and Griffiths (2000); Kryštufek } \\
\text { and Vohralík (2001) }\end{array}$ \\
\hline & & Sorex satunini Ognev, 1922 & $\begin{array}{l}\text { Osborn (1965); Spitzenberger (1968); } \\
\text { Kryštufek and Vohralík (2001) }\end{array}$ \\
\hline & & Suncus etruscus (Savi, 1822) & ÇED (2016) \\
\hline & Talpidae & Talpa levantis Thomas, 1906 & $\begin{array}{l}\text { Çağlar (1971); Felten et al. (1973); } \\
\text { Doğramacı (1988); Doğramacı (1989a); } \\
\text { Doğramacı (1989c); Vohralík (1991); } \\
\text { Kefelioğlu ve Gençoğlu (1996); Kryštufek } \\
\text { and Vohralík (2001) }\end{array}$ \\
\hline
\end{tabular}


Çizelge 2. Devamı.

Table 2. Continue.

\begin{tabular}{|c|c|c|c|}
\hline Takım & Familya & Tür & Literatür kaydı \\
\hline Lagomorpha & Leporidae & Lepus europaeus Pallas, 1778 & Doğramacı (1989c) \\
\hline \multirow[t]{20}{*}{ Rodentia } & Cricetidae & Chionomys nivalis (Martins, 1842) & $\begin{array}{l}\text { Neuhäuser (1936); Osborn (1962); Çağlar } \\
\text { (1967), Felten et al. (1973); Kefelioğlu } \\
\text { (1995); Kryštufek (1999b) }\end{array}$ \\
\hline & & Cricetulus migratorius (Pallas, 1773) & $\begin{array}{l}\text { Osborn (1965); Doğramacı (1989b); } \\
\text { Felten et al. (1971) }\end{array}$ \\
\hline & & Microtus levis Miller, 1908 & $\begin{array}{l}\text { Neuhäuser (1936); Osborn (1962); Çağlar } \\
\text { (1967); Felten et al. (1971); Kefelioğlu } \\
\text { (1995); Yiğit et al. (2006) }\end{array}$ \\
\hline & & $\begin{array}{l}\text { Microtus subterraneus (de Selys- } \\
\text { Longchamps, 1836) }\end{array}$ & $\begin{array}{l}\text { Osborn (1962); Çağlar (1967); Felten et } \\
\text { al. (1971); Kryštufek and Vohralík (2005); } \\
\text { Yiğit et al. (2006) }\end{array}$ \\
\hline & & $\begin{array}{l}\text { Microtus guentheri (Danford and } \\
\text { Alston, 1880) }\end{array}$ & $\begin{array}{l}\text { Kryštufek and Vohralík (2005), ÇED } \\
\text { (2016) }\end{array}$ \\
\hline & & Myodes glareolus (Schreber, 1780) & $\begin{array}{l}\text { Osborn (1962); Çağlar (1967); Felten et } \\
\text { al. (1971); Mursaloğlu (1976); Yiğit et al. } \\
\text { (2006) }\end{array}$ \\
\hline & Gliridae & Dryomys nitedula (Pallas, 1778) & $\begin{array}{l}\text { Osborn (1964); Felten et al. (1973); } \\
\text { Mursaloğlu (1976); Kryštufek and } \\
\text { Vohralík (2005); Yiğit et al. (2003); Yiğit et } \\
\text { al. (2006) }\end{array}$ \\
\hline & & Glis glis (Linnaeus, 1766) & $\begin{array}{l}\text { Osborn (1964); Yiğit et al. (2003); Yiğit et } \\
\text { al. (2006) }\end{array}$ \\
\hline & & $\begin{array}{l}\text { Muscardinus avellanarius (Linnaeus, } \\
\text { 1758) }\end{array}$ & $\begin{array}{l}\text { Kivanç (1983); Kıvanç (1990); Kryštufek } \\
\text { and Vohralík (2005); Yiğit et al. (2006), }\end{array}$ \\
\hline & Muridae & Apodemus flavicollis (Melchior, 1834) & $\begin{array}{l}\text { Doğramacı (1974); Zima and Macholán } \\
\text { (1995); Macholán and Zima (1997); Yiğit } \\
\text { et al. (2006); Kryštufek and Vohralík } \\
\text { (2009) }\end{array}$ \\
\hline & & $\begin{array}{l}\text { Apodemus mystacinus (Danford and } \\
\text { Alston, 1877) }\end{array}$ & $\begin{array}{l}\text { Neuhäuser (1936); Osborn (1965); Felten } \\
\text { et al. (1973); Doğramacı (1974); } \\
\text { Mursaloğlu (1976) }\end{array}$ \\
\hline & & Apodemus uralensis (Pallas, 1811) & $\begin{array}{l}\text { Doğramacı (1974); Zima and Macholán } \\
\text { (1995); Macholán and Zima (1997); Yiğit } \\
\text { et al. (2006), }\end{array}$ \\
\hline & & Apodemus witherbyi (Thomas, 1902) & $\begin{array}{l}\text { Neuhäuser (1936); Osborn (1965a); } \\
\text { Doğramacı (1974); Mursaloğlu (1976); } \\
\text { Macholán and Zima (1997); Yiğit et al. } \\
\text { (2006); Kryštufek and Vohralík (2009) }\end{array}$ \\
\hline & & $\begin{array}{l}\text { Mus macedonicus Petrov and Ruzic, } \\
1983\end{array}$ & $\begin{array}{l}\text { Neuhäuser (1936); Yiğit et al. (2006); } \\
\text { Kryštufek, and Vohralík (2009) }\end{array}$ \\
\hline & & Rattus rattus (Linnaeus, 1758) & $\begin{array}{l}\text { Neuhäuser (1936); Osborn (1964); Yiğgit } \\
\text { et al. (2006); Kryštufek and Vohralík } \\
\text { (2009) }\end{array}$ \\
\hline & & Rattus norvegicus (Berkenhout, 1769) & ÇED (2016) \\
\hline & & Meriones tristrami Thomas, 1892 & ÇED (2016) \\
\hline & Sciuridae & Sciurus anomalus Gmelin, 1778 & $\begin{array}{l}\text { Osborn (1964); Felten et al. (1971); } \\
\text { Mursaloğlu (1976); Kryštufek and } \\
\text { Vohralík (2005) }\end{array}$ \\
\hline & & Sciurus vulgaris Linnaeus, 1758 & ÇED (2016) \\
\hline & Spalacidae & $\begin{array}{l}\text { Nannospalax xanthodon (Nordman, } \\
\text { 1840) }\end{array}$ & $\begin{array}{l}\text { Felten et al. (1973); Matur and Sözen } \\
(2005)\end{array}$ \\
\hline
\end{tabular}

*: LC: Least concern (Asgari endişe), NT: Near threatened (Tehlike sınıında), DD: Data deficient (Veri yetersiz). 
Çizelge 3. Araştırma alanından tespit edilen türlerin korunma statüleri ve lokaliteleri.

Table 3. The conservation status and locality of the species identified from the research area.

\begin{tabular}{|c|c|c|c|c|}
\hline Tür & IUCN* & Cites* & Bern* & $\mathbf{M}$ \\
\hline $\begin{array}{l}\text { Canis aureus } \\
\text { Linnaeus, } 1758\end{array}$ & LC & EK III B & & II \\
\hline $\begin{array}{l}\text { Canis lupus } \\
\text { Linnaeus, } 1758\end{array}$ & LC & EK I B & EK II & II \\
\hline $\begin{array}{l}\text { Vulpes vulpes } \\
\text { (Linnaeus, 1758) }\end{array}$ & LC & & & II \\
\hline $\begin{array}{l}\text { Felis silvestris } \\
\text { Schreber, } 1777\end{array}$ & LC & & EK II & \\
\hline $\begin{array}{l}\text { Lutra lutra } \\
\text { (Linnaeus, 1758) }\end{array}$ & NT & EKI B & EK II & \\
\hline $\begin{array}{l}\text { Martes foina } \\
\text { (Erxleben, 1777) }\end{array}$ & LC & & EK II & \\
\hline $\begin{array}{l}\text { Meles meles } \\
\text { (Linnaeus, 1758) }\end{array}$ & LC & & EK III & 1 \\
\hline $\begin{array}{l}\text { Mustela nivalis } \\
\text { Linnaeus, } 1766\end{array}$ & LC & & EK III & 1 \\
\hline $\begin{array}{l}\text { Ursus arctos } \\
\text { Linnaeus, } 1758\end{array}$ & LC & EK II & EK II & \\
\hline $\begin{array}{l}\text { Capreolus } \\
\text { capreolus } \\
\text { ((Linnaeus, 1758) }\end{array}$ & LC & & EK III & \\
\hline $\begin{array}{l}\text { Sus scrofa } \\
\text { Linnaeus, } 1758\end{array}$ & LC & & EK III & II \\
\hline
\end{tabular}

Rhinolophus

LC

ferrumequinum

(Schreber, 1774)

Pipistrellus

pipistrellus

(Schreber, 1774)

Erinaceus concolor LC

Martin, 1837

LC

Makk* Lokalite

Osmangazi, Uludağ Milli Parkı, Mustafa Kemal Paşa,

Garipçetekke köyü

Orhaneli, Sadağı Kanyonu, Mustafa Kemal Paşa,

Doğanalan köyü, Mustafa Kemal Paşa, Garipçetekke köyü Osmangazi, Uludağ Milli Parkı, Nilüfer Gümüştepe, İnegöl, Cerrah, Orhaneli, Sadağı Kanyonu, Mustafa Kemal Paşa, Doğanalan köyü, Mustafa Kemal Paşa, Garipçetekke köyü, Mustafa Kemal Paşa, Suuçtu Selalesi

Osmangazi, Uludağ Milli Parkı, Nilüfer Gümüştepe, Orhaneli, Sadağı Kanyonu, Mustafa Kemal Paşa, Doğanalan köyü, Mustafa Kemal Paşa, Garipçetekke köyü, Mustafa Kemal Paşa, Suuçtu Şelalesi

Orhaneli, Sadağı Kanyonu

Osmangazi, Uludağ Milli Parkı, Nilüfer Gümüştepe, İnegöl, Cerrah, Orhangazi, Gedelek köyü, Orhaneli, Sadağı Kanyonu, Mustafa Kemal Paşa, Garipçetekke köyü Mustafa Kemal Paşa, Suuçtu Şelalesi

Nilüfer Gümüştepe, İnegöl, Cerrah, Mustafa Kemal Paşa, Doğanalan köyü,

Osmangazi, Uludağ Milli Parkı, Nilüfer Gümüştepe, Mustafa Kemal Paşa, Doğanalan köyü, Mustafa Kemal Paşa, Garipçetekke köyü

Osmangazi, Uludağ Milli Parkı, İnegöl, Cerrah, Mustafa Kemal Paşa, Garipçetekke köyü, Mustafa Kemal Paşa, Suuçtu Şelalesi

Osmangazi, Uludağ Milli Parkı, Orhaneli, Sadağı Kanyonu, Mustafa Kemal Paşa, Doğanalan köyü, Mustafa Kemal Paşa, Suuçtu Şelalesi

Osmangazi, Uludağ Milli Parkı, Nilüfer Gümüştepe, Orhangazi, Gedelek köyü, Orhangazi, Keramet köyü, Karacabey, Kocaçay Deltası, Orhaneli, Sadağı Kanyonu, Mustafa Kemal Paşa, Doğanalan köyü, Mustafa Kemal Paşa, Garipçetekke köyü, Mustafa Kemal Paşa, Suuçtu Şelalesi

Mustafa Kemal Paşa, Doğanalan köyü

EK III

Orhangazi, Gedelek köyü, Orhangazi, Keramet köyü, Mudanya, Kumyaka, Mustafa Kemal Paşa, Doğanalan köyü, Mustafa Kemal Paşa, Garipçetekke köyü

Osmangazi, Uludağ Milli Parkı, Nilüfer Gümüştepe, Gemlik, Umurbey, Orhangazi, Gedelek köyü, Orhangazi, Keramet köyü, Karacabey, Kocaçay Deltası, Mudanya, Kumyaka, Orhaneli, Sadağı Kanyonu, Mustafa Kemal Paşa, Doğanalan köyü, Mustafa Kemal Paşa, Garipçetekke köyü, Mustafa Kemal Paşa, Suuçtu Şelalesi

Osmangazi, Uludağ Milli Parkı, Nilüfer Gümüştepe, Orhangazi, Gedelek köyü, Orhangazi, Keramet köyü, Mudanya, Kumyaka, Mustafa Kemal Paşa, Garipçetekke köyü

Osmangazi, Uludağ Milli Parkı, Nilüfer Gümüştepe, Mudanya, Kumyaka, Orhaneli, Sadağı Kanyonu, Mustafa Kemal Paşa, Doğanalan köyü, Mustafa Kemal Paşa, Garipçetekke köyü,

Orhangazi, Keramet köyü, Orhaneli, Sadağı Kanyonu, Mustafa Kemal Paşa, Doğanalan köyü

Miller, 1908

LC

Muscardinus

LC

EK III 
Çizelge 3. Devamı.

Table 3. Continue.

\begin{tabular}{|c|c|c|c|c|c|}
\hline Tür & IUCN* & Cites* & Bern* & Makk* & Lokalite \\
\hline $\begin{array}{l}\text { Apodemus } \\
\text { flavicollis } \\
\text { (Melchior, 1834) }\end{array}$ & LC & & & & $\begin{array}{l}\text { Osmangazi, Uludağ Milli Parkı, Nilüfer Gümüştepe, } \\
\text { Orhangazi, Keramet köyü, Karacabey, Kocaçay Deltası } \\
\text { Orhaneli, Sadağı Kanyonu, Mustafa Kemal Paşa } \\
\text { Doğanalan köyü, Mustafa Kemal Paşa, Garipçetekke } \\
\text { köyü, Mustafa Kemal Paşa, Suuçtu Şelalesi }\end{array}$ \\
\hline $\begin{array}{l}\text { Mus musculus } \\
\text { Linnaeus, } 1758\end{array}$ & LC & & & & $\begin{array}{l}\text { Osmangazi, Nilüfer Gümüştepe, Gemlik, Umurbey, } \\
\text { Orhangazi, Gedelek köyü, Orhangazi, Keramet köyü, } \\
\text { Nilüfer, Balat Kent Ormanı, Mudanya, Kumyaka, Mustafa } \\
\text { Kemal Paşa, Doğanalan köyü Mustafa Kemal Paşa, } \\
\text { Doğanalan köyü, Mustafa Kemal Paşa, Garipçetekke } \\
\text { köyü }\end{array}$ \\
\hline $\begin{array}{l}\text { Rattus rattus } \\
\text { (Linnaeus, 1758) }\end{array}$ & LC & & & & $\begin{array}{l}\text { Osmangazi, Nilüfer Gümüştepe, Gemlik, Umurbey } \\
\text { Orhangazi, Gedelek köyü, Orhangazi, Keramet köyü } \\
\text { Mudanya, Kumyaka, Nilüfer, Balat Kent Ormanı, Mustafa } \\
\text { Kemal Paşa, Doğanalan köyü, Mustafa Kemal Paşa } \\
\text { Garipçetekke köyü }\end{array}$ \\
\hline $\begin{array}{l}\text { Sciurus anomalus } \\
\text { (Gmelin, 1778) }\end{array}$ & LC & & EK II & & $\begin{array}{l}\text { Osmangazi, Uludağ Milli Parkı, Nilüfer Gümüştepe, } \\
\text { Orhangazi, Gedelek köyü, } \\
\text { Orhangazi, Keramet köyü, Karacabey, Kocaçay Deltası } \\
\text { Mudanya, Kumyaka, Orhaneli, Sadağı Kanyonu, Nilüfer } \\
\text { Balat Kent Ormanı, Mustafa Kemal Paşa, Doğanalan } \\
\text { köyü, Mustafa Kemal Paşa, Garipçetekke köyü, Mustafa } \\
\text { Kemal Paşa, Suuçtu Şelalesi }\end{array}$ \\
\hline $\begin{array}{l}\text { Nannospalax } \\
\text { xanthodon } \\
\text { (Nordman, 1840) }\end{array}$ & DD & & & & $\begin{array}{l}\text { Osmangazi, Uludağ Milli Parkı, Gemlik, Umurbey, } \\
\text { Nilüfer, Balat Kent Ormanı, Mustafa Kemal Paşa, } \\
\text { Doğanalan köyü, Mustafa Kemal Paşa, Garipçetekke } \\
\text { köyü }\end{array}$ \\
\hline
\end{tabular}

*IUCN: The International Union for Conservation of Nature.

${ }^{*}$ Cites: The Convention on International Trade in Endangered Species of Wild Fauna and Flora.

*Bern: Conservation of European Wildlife and Natural Habitats, *MAKK: Merkez Av Komisyonu Kararları.
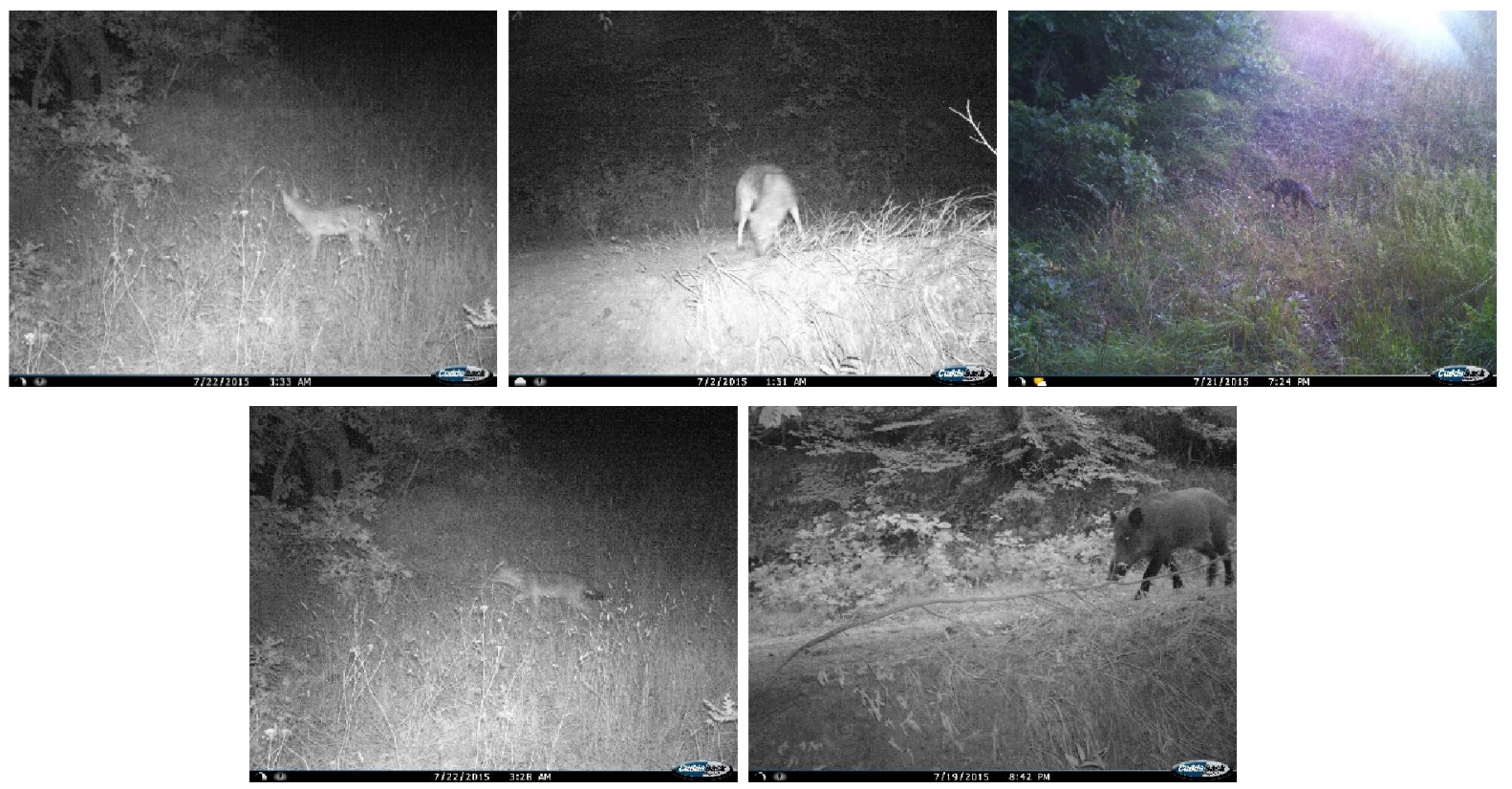

Şekil 3. Fotokapanlarla tespit edilen türlerin görüntüleri (Soldan sağa: Canis aureus, Canis lupus, Felis silvestris, Vulpes vulpes, Sus scrofa).

Figure 3. Images of species detected by wildlife camera traps (From left to right: Canis aureus, Canis lupus, Felis silvestris, Vulpes vulpes, Sus scrofa). 


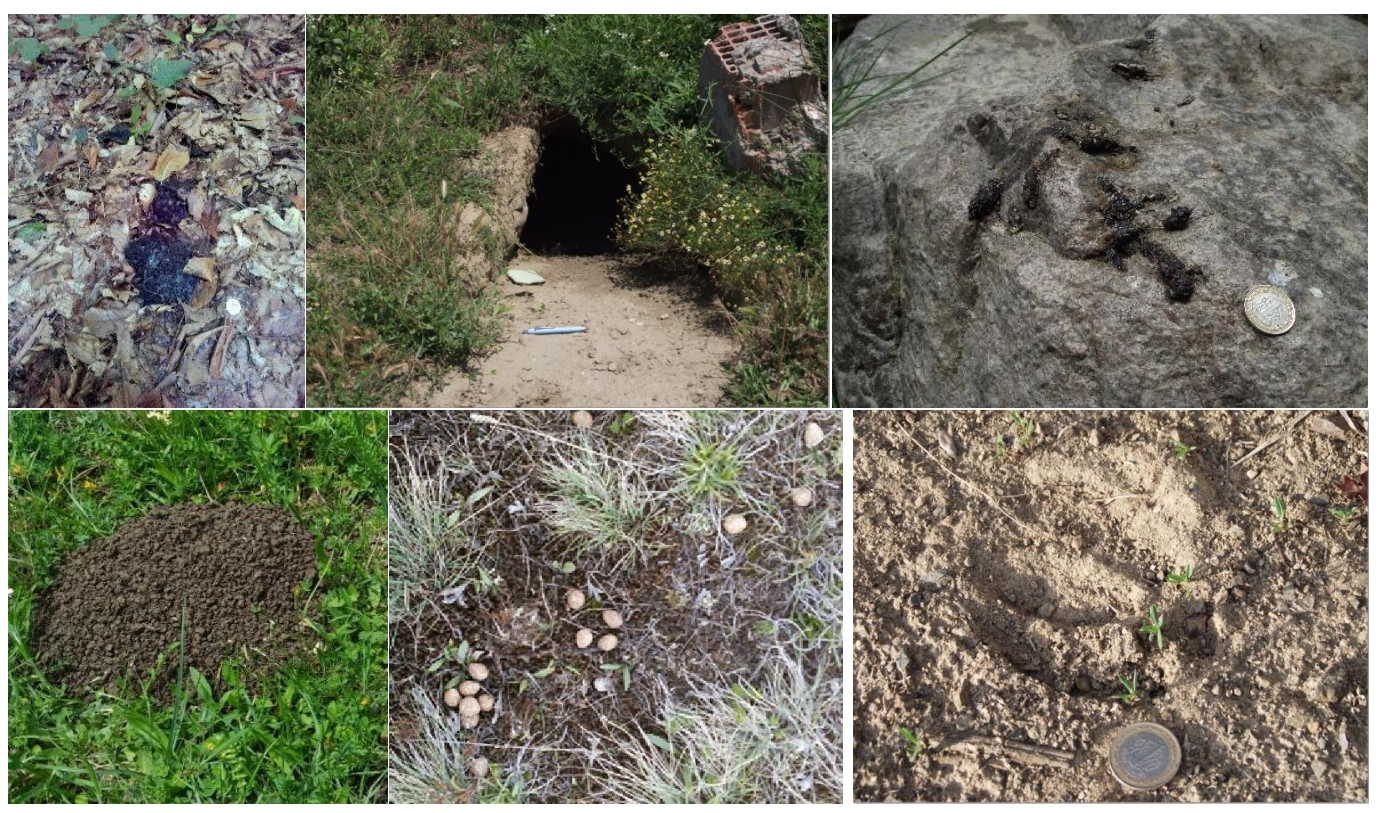

Şekil 4. Arazideki iz, yuva ve dışkı örneklerinden kaydedilen türler (Soldan sağa: Ursus arctos, Meles meles, Lutra lutra, Nannospalax xanthodon, Lepus europaeus, Capreolus capreolus).

Figure 4. Species recorded from the tracks, nests and feces samples in the field (From left to right: Ursus arctos, Meles meles, Lutra lutra, Nannospalax xanthodon, Lepus europaeus, Capreolus capreolus).

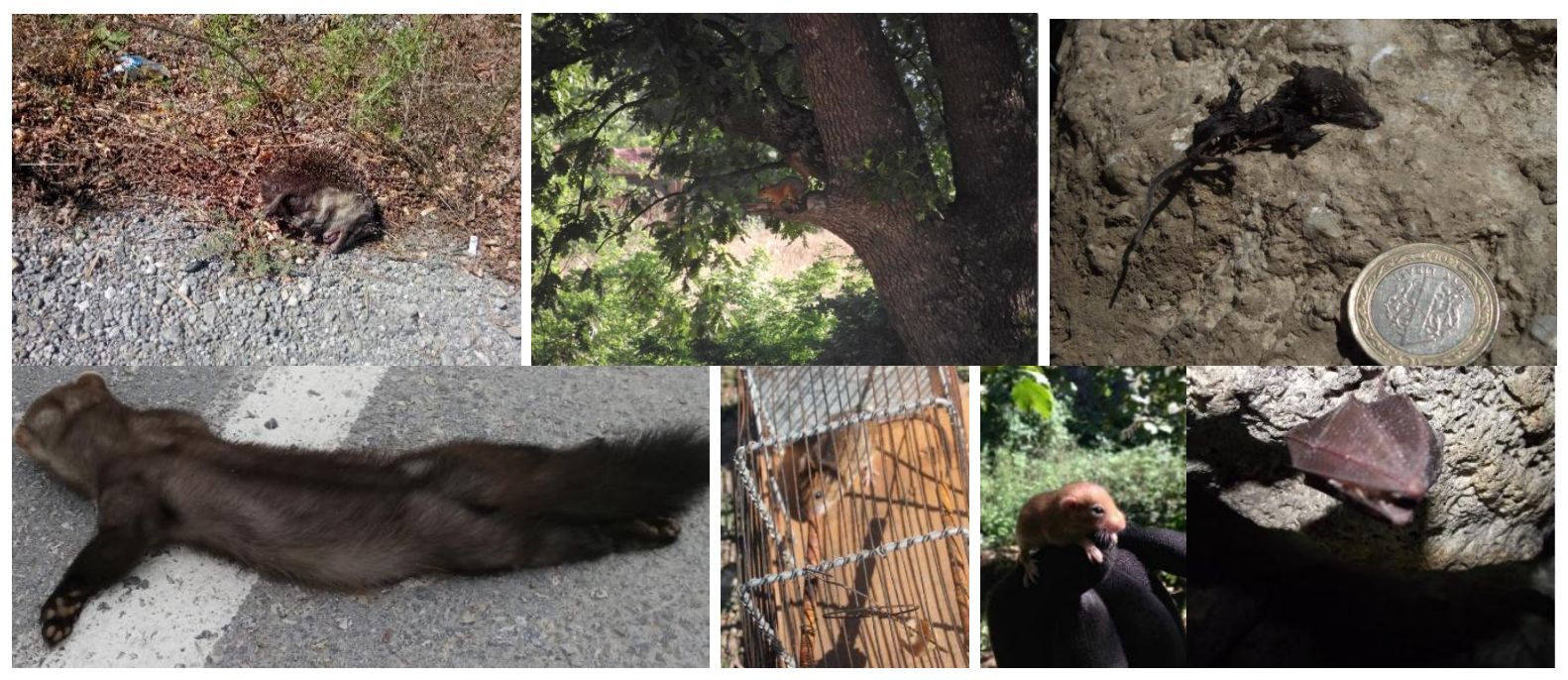

Şekil 5. Doğrudan fotoğrafı kaydedilen türler (Soldan sağa: Erinaceus concolor, Sciurus anomalus, Crocidura leucodon Martes foina, Apodemus flavicollis, Muscardinus avellanarius, Rhinolophus hipposideros).

Figure 5. Directly photographed species (From left to right: Erinaceus concolor, Sciurus anomalus, Crocidura leucodon Martes foina, Apodemus flavicollis, Muscardinus avellanarius, Rhinolophus hipposideros).

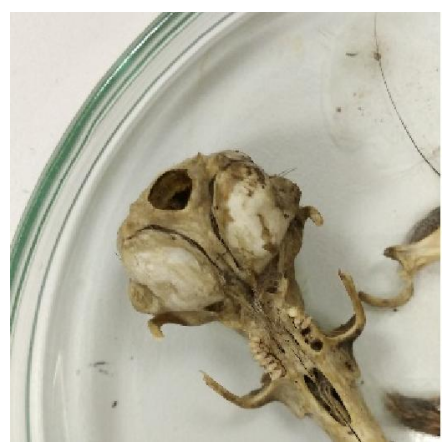

Şekil 6. Peletlerden tespit edilen tür (Microtus levis).

Figure 6. Species determined from pellets (Microtus levis). 
Yerel halk ile yapılan görüşmeler sonucunda Mustafa Kemal Paşa ilçesi, Garipçetekke köyü yakınlarında zehir kullanımı kaynaklı çok sayıda hayvanın telef olduğu tespit edilmiştir. Bu hayvanlar arasında evcil köpekler, tilkiler, kirpiler ve bazı kuş türleri bulunmaktadır (Şekil 7).
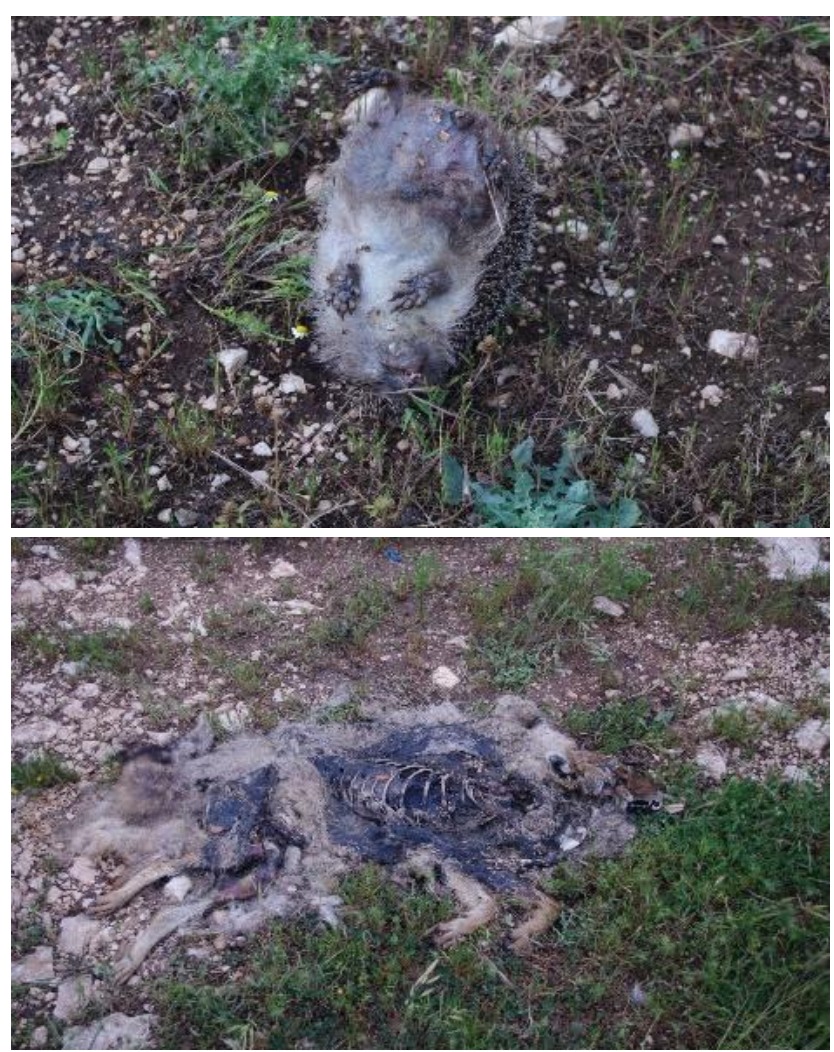

Şekil 7. Garipçetekke köyü arazisinde zehir kullanımı sonucu telef olan memeli örnekleri.

Figure 7. Examples of mammals that are the result of the use of poisons in the Garipçetekke village.

\section{SONUÇ}

Memeli hayvanlar gerek av gerekse avcı olmaları sebebiyle ekosistemin vazgeçilmez elemanlarıdır. Bu sebeple yayılış alanlarının ve popülasyon büyüklüklerinin bilinmesi sürdürülebilir bir dengenin yanında ekonomik anlamda da katkı sağlanmasını gerçekleştirecektir. Arazi çalışmaları ve yerel halk ile yapılan görüşmeler sonucunda elde edilen veriler il genelinde tespit edilen memeli türlerinin maruz kaldığı tehlikelerin başında kaçak avcılık, habitat tahribi ve düzensiz yapılaşmanın bulunduğunu göstermektedir. Özellikle mermer ve taş ocaklarının kurulma safhasından kapatılma safhasına kadar olan süreçte çevreye verdikleri zararın önlenmesi, alanda bulunan yaban hayvanları için hayati önem arz etmektedir. Nesli tehdit altında olması sebebiyle yayılış alanlarını tespit edilmesi çok önemli olan türlerden biri olan Lutra lutra (Su samuru) Orhaneli ilçesi, Sadağı Kanyonu içerisinde tespit edilmiştir. Alanda bulunan Lutra lutra'nın popülasyon yoğunluğunun tespiti, türün koruma ve yönetimi için uygulanması gereken uygun metot konusunda inceleme ve araştırmalara intiyaç duyulmaktadır. Garipçetekke köyü ve çevresi nispeten korunmuş bir alan olup yaban hayvanları için uygun bir alan özelliğinde olduğu düşünülmektedir. Bu konu ile ilgili olarak yerel halkın bilgilendirilmesinin, ekolojik denge ve ekosistem hakkında bilgilendirme toplantıları gibi eğitim faaliyetlerinin yapılmasının, kolluk kuvvetleri ve halkın kaçak avcılık konusunda bilgilendirilmesi ve önlenmesi için ortak çalışma yapabilmelerinin sağlanmasının faydalı olacağı düşünülmektedir.

\section{TEŞEKKÜR}

Bu araştırma Çevre ve Şehircilik Bakanlığı́nın "Bursa ve Yalova illeri Doğal Sit Alanlarının Ekolojik temelli Bilimsel Araştırma Projesi" kapsamında Tabiat Varlıklarını Koruma Şube Müdürlüğü bilgisi dahilinde yapılmış olup Kurum çalışanlarından Şube Müdürü Sayın Levent Kül'e ve çalışma arkadaşlarına, arazi çalışmaları esnasında ve sonraki süreçte verdikleri destekten ötürü teşekkür ederim.

\section{KAYNAKLAR}

Albayrak İ., 1993. Batı Türkiye yarasaları ve yayılışları (Mammalia: Chiroptera). Doğa-Turkish Journal of Zoology, 17(3): 237-257.

BERN 1979. Convention on the Conservation of European Wildlife and Natural Habitats. European Treaty SeriesNo. 104. Bern. http://www.coe.int/en/web/bernconvention/ [Access: June 9, 2017].

Benda P and Horáček I., 1998. Bats (Mammalia: Chiroptera) of the Eastern Mediterranean. Part 1. Review of distribution and taxonomy of bats in Turkey. Acta Societatis Zoologicae Bohemicae, 62(4): 255-313.

Çağlar M., 1961. Küçük nalburunlu yarasa (Rhinolophus hipposideros) hakkında, Türk Biologi Dergisi, 11: 11-13.

Çağlar M., 1965. Chiropterenfauna der Türkei. -Türkiye'nin Chiroptera favnası. İstanbul Üniversitesi Fen Fakültesi Mecmuası B, 30(3-4): 125-134.

Çağlar M., 1967. Türkiye'nin Gömülgen fare (Microtin)leri. Türk Biologi Dergisi, 17(4): 103-118.

Çağlar M., 1968. Türkiye'nin Yarasaları I. Türk Biologi Dergisi, 18(1): 5-18.

Çağlar M., 1971. Türkiye'nin Köstebek (Talpa) türleri. Mole (Talpa) species of Turkey. Türk Biologi Dergisi, 21(1-4): 123-126.

ÇED 2016. T.C Bursa ili 2015 yılı Çevre Durum Raporu. Bursa Valiliği Çevre ve Şehircilik II Müdürlüğü, ÇED ve Çevre 
İzinleri Şube Müdürlüğü, Bursa.

CITES 2015. The Convention on International Trade in Endangered Species of Wild Fauna and Flora. Appendices I, II and III, http//www.cites.org. [Access: February 5, 2015].

Doğramacı S., 1974. Türkiye Apodemus (Mammalia: Rodentia)'larının Taksonomik Durumları. T.C. Gıda, Tarım ve Hayvancılık Bakanlığı Zirai Mücadele Müdürlüğü Araştırma Eserleri Serisi.

Doğramacı S., 1988a. Türkiye Akdeniz köstebeği Talpa caeca (Mammalia: Insectivora)'nın coğrafik varyasyonları. Ondokuz Mayıs Üniversitesi Ziraat Fakültesi Dergisi, 3 (2): 207-222.

Doğramacı S., 1988b. Türkiye Talpa (Mammalia: Insectivora) türlerinin ayırd edilmesinde pelvisin önemi. DoğaTurkish Journal of Zoology, 13(2): 67-76.

Doğramacı S., 1989a. Türkiye Köstebeklerinin (Cins: Talpa: Mammalia) taksonomisi ve yayılışları. Doğa-Turkish Journal of Zoology, 13(3): 204-219.

Doğramacı S., 1989b. Türkiye Cricetulus migratorius (Mammalia: Rodentia) türünün coğrafik varyasyonları. Ondokuz Mayıs Üniversitesi Fen Dergisi, 1(3): 1-24.

Doğramacı S., 1989c. Türkiye memeli faunası. Ondokuz Mayıs Üniversitesi Fen Dergisi, 1(3): 107-136.

Felten H., Spitzenberger F und Storch G., 1971. Zur Kleinsäugerfauna West-Anatoliens. Teil I, Senckenbergiana Biologica, 52(6): 393-424.

Felten H., Spitzenberger $F$ und Storch G., 1973. Zur Kleinsäugerfauna West-Anatoliens. Teil II, Senckenbergiana Biologica, 54(4-6): 227-290.

Felten H., Spitzenberger $F$ und Storch G., 1977. Zur Kleinsäugerfauna West-Anatoliens. Teil IIIa, Senckenbergiana Biologica, 58: 1-44.

Huş S ve Göksel E., 1981. Türkiye av hayvanlarının yayılış yerleri. İstanbul Üniversitesi Orman Fakültesi Dergisi B, 31(2): 68-81.

IUCN 2017. The International Union for Conservation of Nature (ver. 3.1.) http://www.iucnredlist.org [Access: June 9, 2017].

IÇDR 2011. Bursa Valiliği Çevre ve Şehircilik İ Müdürlüğü, Bursa İl Çevre Durum Raporu.

Karataş A and Sözen M., 2004. Contributions to karyology, distribution and taxonomic status of the long-winged bat, Miniopterus schreibersii (Chiroptera: Vespertilionidae), in Turkey. Zoology in the Middle East, 33: 51-64.

Kahmann H ve Çağlar M., 1960, Türkiye'de memeli hayvanlar araştırımı sahasında yeni buluşlar. Türk Biologi Dergisi, 10(3): 119-126.

Kasparek M., 1988. On the occurrence of the Weasel, Mustela nivalis, in Turkey. Zoology in the Middle East, 2: 8-11.
Kefelioğlu H., 1995. Türkiye Microtus (Mammalia: Rodentia) cinsinin taksonomisi ve yayılışı. Turkish Journal of Zoology, 19(1): 35-63.

Kefelioğlu H ve Gençoğlu S., 1996. Karadeniz Bölgesi Talpa (Mammalia: Insectivora)'larının taksonomisi ve yayılışı. Turkish Journal of Zoology, 20(1): 57-66.

Kefelioğlu H and Tez C., 1999. The distribution problem of Crocidura russula (Hermann 1780) (Mammalia: Insectivora) in Turkey. Turkish Journal of Zoology, 23(3): 247-251.

Kıvanç E., 1983. Die Haselmaus, Muscardinus avellanarius L., in der Türkei. Bonner zoologische Beiträge, 34(4): 419428.

Kıvanç E., 1990. Fındık faresinin (Muscardinus avellanarius Linnaeus, 1758) Türkiye'deki üreme biyolojisi. Journal of Biology Faculty of Science and Arts of Gazi University, 1: 31-41.

Kryštufek B., 1999. Snow voles, genus Chionomys, of Turkey. Mammalia, 63: 323-339.

Kryštufek B and Griffiths HI., 2000, Cranial differentiation in Neomys water shrews. Folia Zoologica, 49(2): 81-87.

Kryštufek B and Vohralík V., 2001. Mammals of Turkey and Cyprus, Volume 1: Introduction, Checklist, Insectivora. Knjiznica Annales Majora, Koper.

Kryštufek B and Vohralík V., 2004. Molar size variation in three species of Pine Vole in Asia Minor: Microtus subterraneus, M. majori, and M. daghestanicus (Rodentia: Arvicolinae). Israel Journal of Zoology, 50(4): 311-319.

Kryštufek B and Vohralík V., 2005. Mammals of Turkey and Cyprus, Volume 2, Rodentia I: Sciuridae, Dipodidae, Gliridae, Arvicolinae, Knjiznica Annales Majora, Koper.

Kryštufek B and Vohralík V., 2009. Mammals of Turkey and Cyprus, Volume 3, Rodentia II: Cricetinae, Muridae, Spalacidae, Calomyscidae, Capromyidae, Hystricidae, Castoridae, Knjiznica Annales Majora, Koper.

Kumerloeve H., 1967. Zur Verbreitung Kleinasiatischer Raubund Huftiere sowie eineger Großnager. Sonderdruck aus Säugetierkd Mitt., 15(4): 337-409.

MAKK 2016. Merkez av komisyonu kararları. http://www.resmigazete.gov.tr/eskiler/2015/06/2015060 5M1-1.pdf [Erişim: 9 Haziran 2017].

Macholán M and Zima J., 1997. Absence of the B chromosomes in karyotypes of the yellow-necked wood mouse (Apodemus flavicollis) from Asia Minor. Folia Zoologica, 46(2): 191-192.

Matur F and Sözen M., 2005. A karyological study on subterrranean mole rats of the Spalax leucodon Nordmann, 1840 (Mammalia: Rodentia) superspecies in northwestern Turkey. Zoology in the Middle East, 36: 510.

MGM 2017. Bursa ilinin meteorolojik verileri. Meteoroloji 
Genel Müdürlüğü https://www.mgm.gov.tr/ veridegerlendirme/il-ve-ilceler-istatistik.aspx?m=BURSA [Erişim: 9 Haziran 2017].

Mursaloğlu B., 1976. Türkiye kemiricileri (Mammalia: Rodentia), TÜBiTAK, Matematik, Fiziki ve Biyolojik Bilimler Araştırma Grubu, Proje No: TBAG-52 ve Ek Proje No: TBAG-99, Ankara.

Musser GG., Carleton MD., 2005. Mammals Species of the World A Taxonomic and Geographic Reference. Johns Hopkins University Press.

Neuhäuser G., 1936, Die Muriden von Kleinasien. Zeitschrift für Säugetierkunde, 11: 161-236.

Osborn DJ., 1962. Rodents of the subfamily Microtinae from Turkey, Journal of Mammalogy, 43: 515-529.

Osborn DJ., 1964. The hare, porcupine, beaver, squirrels, jerboas and dormice of Turkey. Mammalia, 28(4): 573592.

Osborn DJ., 1965. Rodents of the subfamilies Murinae, Gerbillinae and Cricetinae from Turkey. The Journal of the Egyptian Public Health Association Kahiro, 40(5): 401-424.

Osborn DJ., 1965b. Hedgehogs and shrews of Turkey. Proceedings of the United States National Museum, Smithsonian Inst., Washington, DC., 117: 553-566.

Sert H., Yağcı Ş., Aktaş M ve Karaer Z., 2001. Türkiye'nin farklı bölgelerinden yakalanan kemiricilerde kene (Acari: Ixodidae, Argasidae) enfestasyonu. Acta Parasitologica Turcica, 25(3): 280-282.

Spitzenberger F., 1968. Zur Verbreitung und Systematik Türkischer Soricinae (Insectivora: Mamm.), Annales des naturhistorischer Museum Wien, 72: 273-289.

Spitzenberger F., 1970. Zur Verbreitung und Systematik Türkischer Crocidurinae (Insectivora: Mammalia),
Annales des naturhistorischer Museum Wien, 74: 233252.

Şimşek N., 1979. Türkiye Crocidura (Mammalia: Insectivora)'larının Taksonomik Durumları ve Yayılışları. Doktora Tezi (Basılmamış), Ankara Üniversitesi Fen Fakültesi, Ankara.

Şimşek N., 1980. Türkiye Crocidura suaveolens (Pallas, 1811) ve Crocidura russula (Hermann, 1780)'Iarının (Mammalia: Insectivora) Diskriminant Analiz Yöntemi ile Ayırt Edilmesi. TÜBiTAK VII. Bilim Kongresi, 6-10 Ekim 1980, Aydın.

Tez C., 2000. Taxonomy and distribution of the Whitetoothed shrews (Crocidura) (Soricidae: Insectivora: Mammalia) of Turkey. Turkish Journal of Zoology, 24(4): 365-374.

Vohralík V., 1991. A record of the mole Talpa levantis (Mammalia: Insectivora) in Bulgaria and the distribution of the species in the Balkans. Acta Universitatis Carolinae Biologica, 35(3-4): 119-127.

von Helversen O., 1989. New records of bats (Chiroptera) from Turkey. Zoology in the Middle East, 3: 5-18.

Yarar M ve Magnin G., 1997. Türkiye'nin Önemli Kuş Alanları. Doğal Hayatı Koruma Derneği, İstanbul.

Yiğit N., Çolak E., Çolak R., Özkan B and Özkurt Ş., 2003. On the Turkish populations of Dryomys nitedula (Pallas 1779) and Dryomys laniger (Felten and Storch, 1968) (Mammalia: Rodentia). Acta Zoologica Academiae Scientiarum Hungaricae, 49(1): 147-158.

Yiğit N., Çolak E., Sözen M and Karataş A., 2006. Rodents of Türkiye "Türkiye Kemiricileri". Meteksan AŞ, Ankara.

Zima J and Macholán M., 1995. B chromosomes in the wood mice (Genus: Apodemus). Acta Theriologica, 3: 75-86. 